\title{
Comparison of lithium niobate and silicon substrate on phase shift and efficiency performance for mach-zehnder interferometer modulator
}

\author{
Nor Hidayah Roslan', Aziati H. Awang ${ }^{2}$, Mohd Hanapiah M. Yusoff ${ }^{3}$, Ahmad Rifqi Md Zain ${ }^{4}$ \\ ${ }^{1,2}$ Faculty of Electrical Engineering, Universiti Teknologi MARA (UiTM), Malaysia \\ ${ }^{3}$ Faculty of Applied Sciences, Universiti Teknologi MARA (UiTM), Malaysia \\ ${ }^{4}$ Institute of Microengineering and Nanoelectronics (IMEN), Universiti Kebangsaan Malaysia (UKM), Malaysia
}

\begin{abstract}
Article Info
Article history:

Received Jun 1, 2020

Revised Aug 10, 2020

Accepted Sep 25, 2020

\section{Keywords:}

Group velocity

Lithium niobate

Mach-zehnder interferometer

Optical modulator

Slow-light

ABSTRACT

In this study, the low-group velocity slow-light mach-zehnder interferometer (MZI) modulator, low loss and high efficiency for two modulator substrate lithium niobate $(\mathrm{LN})$ and silicon were presented and optimized at $1.55 \mu \mathrm{m}$ operating wavelength. The high power consumption of conventional modulator was the major drawback in the operation of modulators. Therefore, it was a good time for low-power modulator design and development and to compare the LN and Silicon modulator on the phase shifted using the slow-light technique by designing the full MZI modulator consisting of splitter and combiner on both substrates. The phase shift of LN is $2 \%$ compared with the silicon $0.09 \%$ and higher phase shift give better performance with low power consumption due to the change of modulating voltage of the MZI modulator for $\mathrm{LN}$ while the silicon depends on modulating voltage manipulating concentration of charge carrier in doped silicon.
\end{abstract}

This is an open access article under the CC BY-SA license.

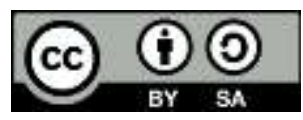

\section{Corresponding Author:}

Aziati Husna Hj Awang

Department of Electrical Engineering

Universiti Teknologi MARA (UiTM)

40450 Shah Alam, Selangor Darul Ehsan, Malaysia

Email: aaziatihusna@gmail.com

\section{INTRODUCTION}

Nowadays, there has been rapid and fast enhancement of the computer chip performance and a reduction in individual components size. However, most electrical interconnects, which carry data inside the microprocessors and between the processor and memory have drawbacks of high loss, low efficiency and high power consumption [1,2]. Traditional optical interconnect systems consist of optical waveguide and optic-electric/electro-optic (OE/EO) conversion devices, in order to achieve the multi-level modulation formats which are commonly used for high-speed transmission systems. Thus, accuracy of OE conversion is very important as well as the efficiency of conversion and response time of such device [3-5]. However, optical interconnects still remains difficult to be optimized, due to the lack of suitable material and modulation mechanisms [6]. Therefore, in this paper we discuss the design of the photonic crystal waveguide with different substrate of lithium niobate (LN) and silicon materials.

The LN electro-optic modulators have been the optoelectronic industry's workhorse for the decades [7-9], however, it is difficult to be integrated on-chip due to difficulties and complications in the fabrication process. Currently, disadvantages of LN modulators are bulky, costly, restricted bandwidth, high drive voltages and also unable to achieve the full potential of materials [10-12]. Future of the photonic systems 
requires modulators with a CMOS-compliant drive voltage, large bandwidth, low insertion loss, excellent signal quality and compatibility with large-scale manufacturing. Although the material of LN is difficult to incorporate there are other photonic material that are compatible with the fabrication process such as silicon, which have good potential for integration with CMOS electronics [13-15], for example low drive voltages and high bandwidth polymers $[16,17]$. A main material for high-speed EO conversion in modulator is silicon mach-zehnder optical modulators $[18,19]$.

Nowadays, the system operates at bit rates that surpass bit rates of $100 \mathrm{~Gb} / \mathrm{s}$. The optical signal is demanded to have high speed and bandwidth for future and potential networks [20, 21]. Some developments of optical networks with the use of the mach-zehnder interferometer (MZI) modulator were presented [22]. MZI is the device that used to calculate the relative phase shift between two beams of light source either by changing the length of one arm or by putting a sample in one the arm by biasing due to high power consumption that needs to be removed. The effects of the high power in the MZI modulator can lead to high group velocity and losses. One at the input acts as a splitter and at the output acts as a combiner. At the output, the light is split into two and merges. The optical path length of two arms allows the phase shifted according to delay to into the wavelength of the input signal. This property is used to design the number of optical devices used in the all-optical domain for signal processing [23]. In previous research the Y-structure show the phase shift of $2.08 \mathrm{rad}$ [24]. Higher phase shift gives a better performance of the device. This research shows higher phase shift by numerically analyze the the phase shift of the device, and the transmission efficiency of the combiner is above $70 \%$ with the step taper [25]. The coupling efficiency was enhanced and the transmission of the modulator was improved.

In this article, there was a theoretical study of two types of material of the MZI modulator and photonic crystal waveguide (PCW) that are LN and silicon. Both modulators worked at $1.55 \mu \mathrm{m}$ of communication wavelength. For these designs we were able to examine the slow light and the group velocity in both PCW of different materials and the phase shift of one arm of MZI by the variation of the refractive index, RI. In addition, the simulation results showed that the enhancement and improvement for both of MZI modulators with different types of material and the phase shifted has been improved to achieve better performance of the device. The MZI modulator can be commonly used in on-chip for the optical interconnect applications and as well as for the embedded devices for example in photonic crystal cavities.

\section{RESEARCH METHOD}

Figure 1(a) shows the full schematic and Figure 1(b) schematic design of the MZI modulator of LN photonic crystal cavities consisting of three parts which are splitter, PCW and combiner. By etching the air holes, the photonic crystal waveguide is structured. L represents the length of the modulator and the total length of the modulator is L1+2L2. The length of the splitter and combiner are the same length. Both the Y shape and the waveguide bend are built for the splitter and combiner parts. The slow light method is applied by both arms of the MZI modulator via the L1 length of the slow light waveguides. All the three electrodes are located in the waveguide line of slow light, these electrodes are similar to the waveguides of slow light, and there is an EO response effect on LN. The voltage, $\mathrm{U}$ is applied to the center electrode that can produce a voltage wave. Both external electrodes are to the ground. The incident light is polarized transverse electric (TE). The waveguide is designed to eliminate one row of the air holes in the perfect PCW for both of the materials that are $\mathrm{LN}$ and silicon as in Figure 2. The photonic crystal waveguide holes radius $\mathrm{R}=0.32 \mathrm{a}$ the thickness of the slab, $h=362 \mathrm{~nm}$ and lattice constant, $a=0.654$. The optimization of the MZI modulator can be seen in the following parts for both materials.

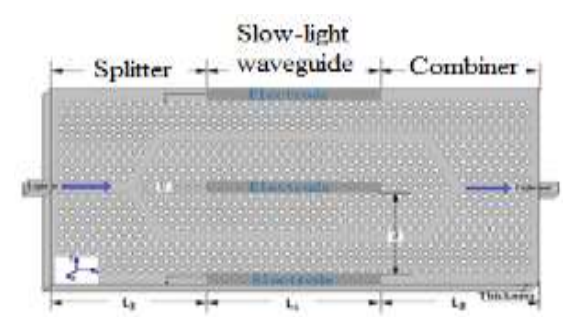

(a)

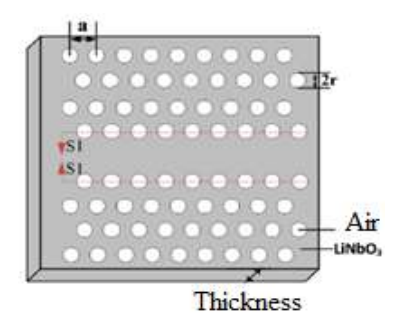

Thickness

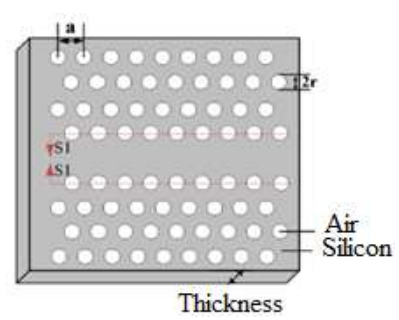

(b)

Figure 1. (a) The full schematic design of the LiNbO3 photonic crystal cavitiey-based MZI modulator, (b) The schematic design of the PCW slow-light implementation for both LiNbO3 and silicon materials 
For both PCW models with the various materials, we introduce slow light techniques in the photonic crystal waveguide. According to the optical theory the group index ng of slow light, showing the slowdown factor compared to the speed of light in vacuum $\mathrm{c}$, is given by $\mathrm{ng}=\mathrm{c} / \mathrm{vg}=\mathrm{c} \mathrm{dk} / \mathrm{d} \omega$ where $\mathrm{k}$ and $\omega$ are the wave number and angular frequency respectively [26, 27]. This means that the slow light is due to the large firstorder dispersion $\mathrm{dk} / \mathrm{d} \omega$. Furthermore, the slow light is limited by the delay-bandwidth product written as $\operatorname{ng}(\Delta \omega / \omega)=n g(\Delta \lambda / \lambda)=\Delta \mathrm{n}$, where $\Delta \omega$ and $\Delta \lambda$ are the frequency and wavelength bandwidth of slow light, respectively and $\Delta \mathrm{n}$ is the effective refractive index change in material or structure within the bandwidth [28, 29]. This relationship implies that, one cannot expect a large ng independently of the bandwidth. If one is considering any application of slow light, the bandwidth needed for the target application should first be calculated and the maximum ng from the above equation estimated.

\section{RESULTS AND DISCUSSION}

The complete of TE propagation information of the perfect photonic crystal for LN is shown in Figure 2(a). It shows the existing band gap between 0.39 and 0.46 below the light line and is determined to solve the Maxwell equations using the plane wave expansion (PWE) technique. Figure 2(b) is the measured band gap for silicon which is in between 0.25 and 0.38 . The photonic band gap of the main propagation mode is shown in Figure 2(c) by the (blue line) of the signal line defect PCW, which supports the lossless even mode. The slow light of PCW support a low group velocity, $\mathrm{Vg}$ where a flat group velocity dispersion region. However, left out the dispersion of slow light in LN can cause the signal waveform to be influenced by excessive group velocity dispersion and signal distortion. It can also affect the performance for both PCW materials in optical interconnect applications. The implication changes the radius of the holes of PCW to the amount of the flat signal of low group velocity dispersion region. The band diagram and the gap between 0.36 and 0.46 when the $\mathrm{r}=0.32 \mathrm{a}$ and $\mathrm{a}=0.6$ is shown as in Figure 2(c).

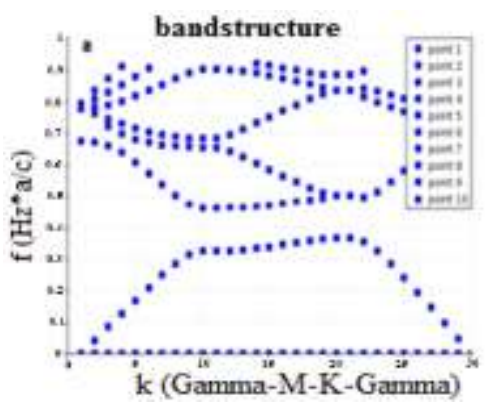

(a)

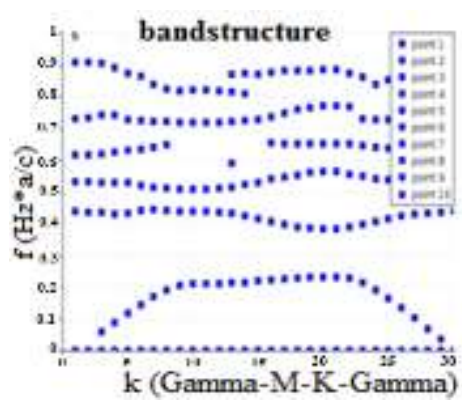

(b)

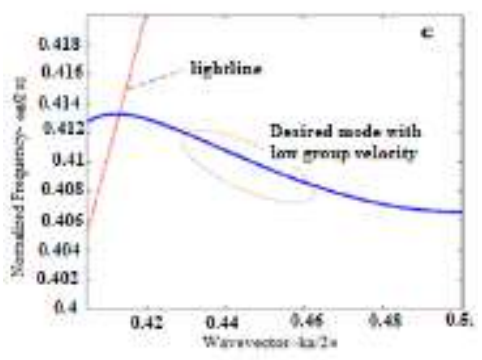

(c)

Figure 2. (a) and (b) The computational bandstrucuture diagram for the perfect LiNbO3 of PCW structure, (c) The computational bandstructure diagram for the perfect Silicon of PCW structure

$$
\Delta n=-\frac{n^{3} L N \gamma_{33} U}{2 d}
$$

Where $\mathrm{nLN}$ the extraordinary refractive index, RI which represents the nonlinear EO coefficient, $\mathrm{U}$ is the applied voltage, and $\mathrm{d}$ is for the gap between the electrodes. To measure the phase shift this depends on the applied voltage;

$$
\Delta \phi=\frac{2 \pi}{\lambda} \cdot \Delta n \cdot L
$$

Where $\lambda$ is the wavelength, $\Delta \mathrm{n}$ represents the index change, and L refers to the length of the region with which the electrical field is applied. When the applied voltage is applies differently so that the index changes also change, such that the calculated refractive index changes also change. If the refractive index changes the simulation results show that even with a small change, the group velocity and loss change. The group velocity modifies the slow light technique occur and give the phase shifted. The MZI modulator has 
two arms, in order to determine the refractive index both arm using the formula where for the upper arm $\mathrm{n}_{\mathrm{LN}}$ $|\Delta n|$ and the lower arm $n_{\mathrm{LN}}+|\Delta n|$, when $|\Delta n|$ is 0.00331 for $0.66 \mathrm{~V}$ and $|\Delta n|$ is 0.00662 is for $1.32 \mathrm{~V}$, the simulation results as shown Figure 2. The phase shift for $0.66 \mathrm{~V}$ is $1.22892 \mathrm{rad}$ while for the applied voltage of $1.32 \mathrm{~V}$ is $2.45784 \mathrm{rad}$. Other properties of the mode, suach as group velocity and loss, can also be determined in addition to calculating the band structure. As the group velocity reduces, the loss increases. $\mathrm{R}=0.32 \mathrm{a}=0.192$, and $\mathrm{a}=0.6$. The refractive index for the applied voltage of $0.06 \mathrm{~V}$ is $\mathrm{n}=2.13969$ and the refractive index of $1.32 \mathrm{~V}$ is $\mathrm{n}=2.13638$, both of which are determined using the formula in Figure 3.

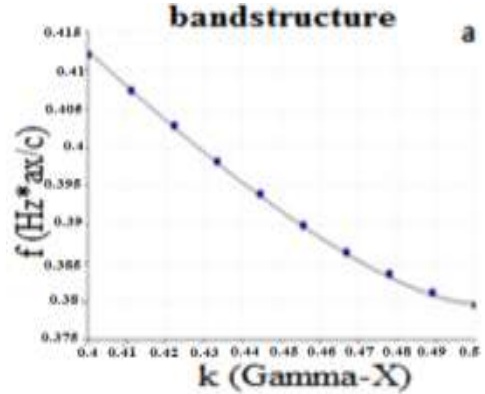

(a)

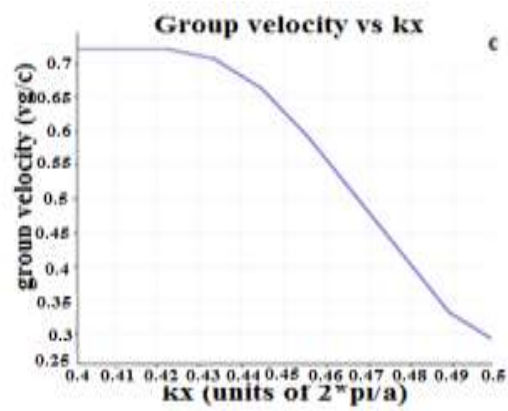

(c)

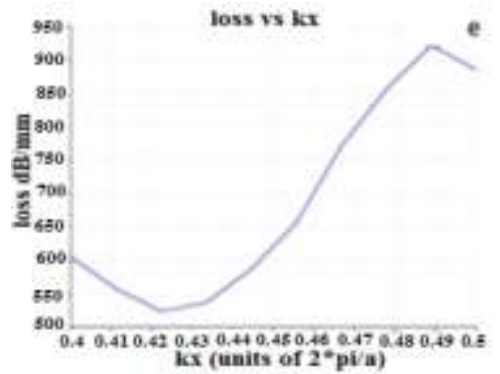

(e)

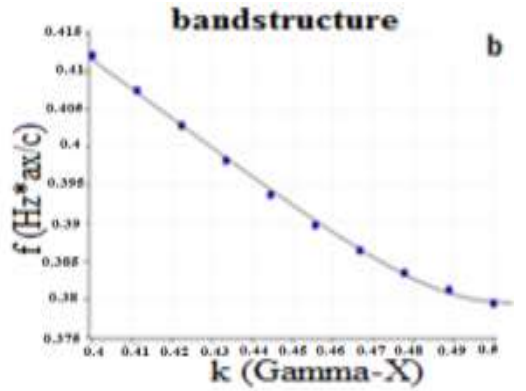

(b)

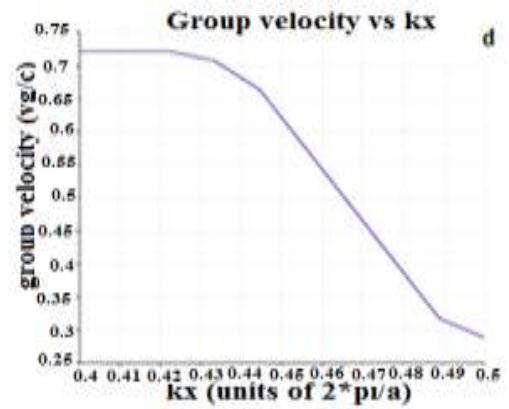

(d)

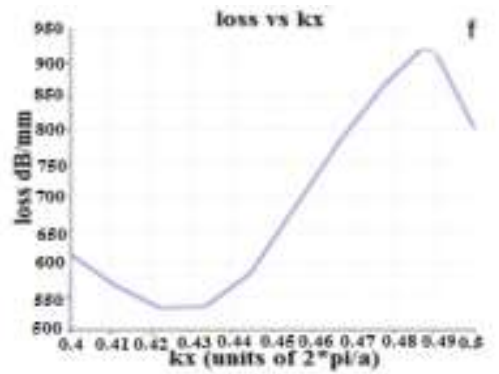

(f)

Figure 3. The measured effects of the LN simulation with variuos voltages. (a), (c), (e) with respect to 0.06V and (b), (d), (f) with respect to $1.32 \mathrm{~V}$

The modulation principle for the silicon material as shown in Figure 4 is based on the free carrier dispersion effect and the relationship between the index perturbation and carrier concentration perturbation by the following function when the wavelength of incident light $\lambda=1.55 \mu \mathrm{m}$.

$$
\Delta \mathrm{n}=\Delta \mathrm{ne}+\Delta \mathrm{nh}=-\left[8.8 \times 10^{-22}(\Delta \mathrm{Ne})+8.5 \times 10^{-18}(\Delta \mathrm{Nh}) 0.5\right]
$$

$\Delta$ ne and $\Delta \mathrm{nh}$ are the refractive index changes caused by the changes of the electrons and holes concentrations respectively. $\Delta$ ne and $\Delta$ nh are the changes of electron and hole concentrations respectively. The change of the refractive index will measure the refractive index for both arm of the MZI modulator where for the upper arm nsi $-|\Delta n|$ and for the lower arm nsi $+|\Delta n|$ where nsi isthe refractive index of silicon. Then we try the different of carrier concentrations for holes and electrons $(\Delta \mathrm{Ne}) \mathrm{c}=1 \times 10^{15} \mathrm{~cm}^{-2}$ and 
$(\Delta \mathrm{Nh}) \mathrm{c}=4 \times 10^{15} \mathrm{~cm}^{-2}$ and for the second carrier concentration is $(\Delta \mathrm{Ne}) \mathrm{c}=(\Delta \mathrm{Nh}) \mathrm{c}=3 \times 10^{17} \mathrm{~cm}^{-3}$ From the change of the refractive index we can calculate the phase shifted by this formula, $\Delta \emptyset=2 \pi / \lambda$. $\Delta n$. $L$. The phase different for the first concentration after the measurement $\Delta \emptyset$ is 0.00989 and the second is 0.09802 . The first phase different is smaller than the second phase different. This is because the phase different depends on the carrier concentrations.

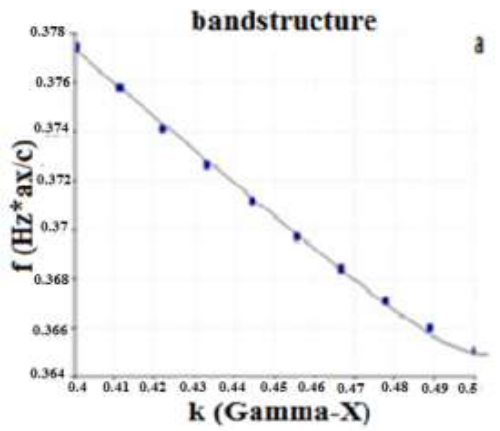

(a)

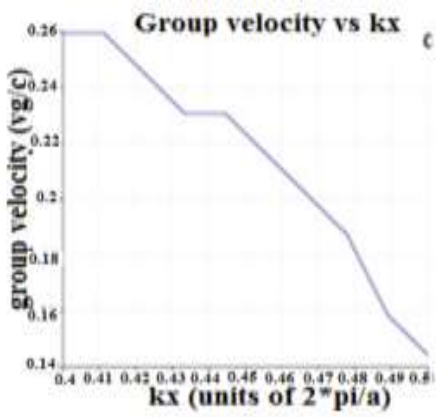

(c)

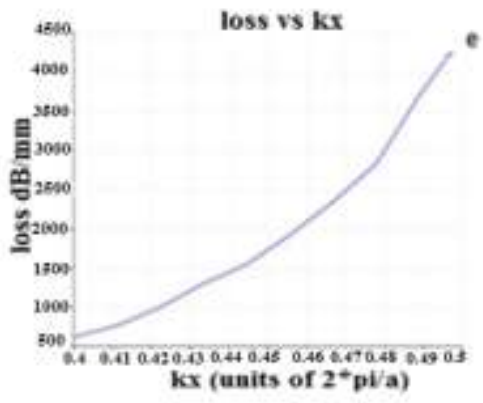

(e)

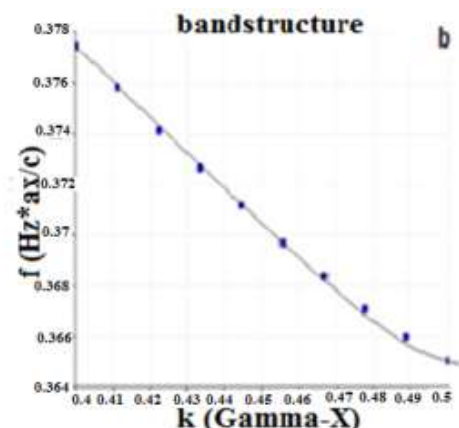

(b)

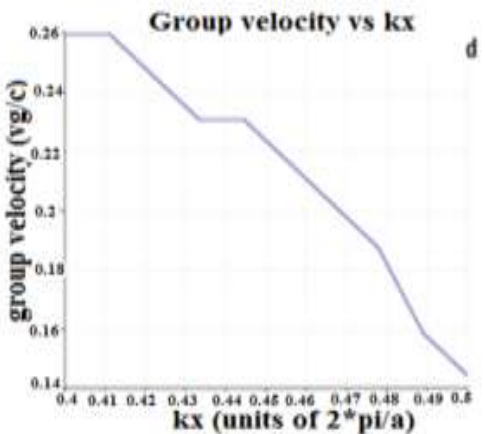

(d)

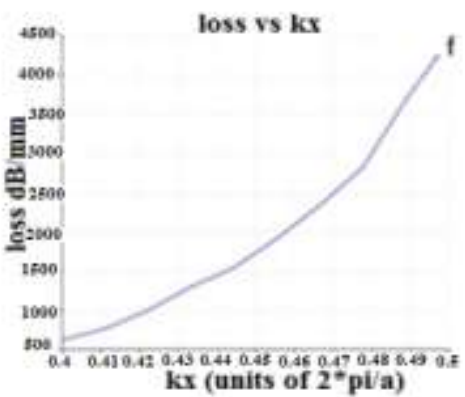

(f)

Figure 4. The results show the different of the carrier concentration for the silicon. (a), (c), (e) for $(\Delta N e) c$ $=1 \times 1015 \mathrm{~cm}-2$ and $(\Delta \mathrm{Nh}) \mathrm{c}=4 \times 1015 \mathrm{~cm}-2$ and $(\mathrm{b}),(\mathrm{d}),(\mathrm{f})$ for $(\Delta \mathrm{Ne}) \mathrm{c}=(\Delta \mathrm{Nh}) \mathrm{c}=3 \times 1017 \mathrm{~cm}-3$

Figure 5 the optimized schematic of photonic crystal splitter-combiner with the designated holes determines the holes where the radius or position changes. The lattice constant of $\mathrm{LN} \mathrm{a}=0.6$ and $\mathrm{r}=0.192$ while for silicon $a=0.5$ and $r=0.2$. In order to obtain the good and better efficiency, the radius and position of holes are selected. Figure 6 shows the steady state electric field figure for TE-polarized wave propagation through an optimized splitter configuration at $1.55 \mu \mathrm{m}$ wavelength where the wavelength range is between $1.43-1.67 \mu \mathrm{m}$ and the band gap for the combiner of the MZI modulator with the same of lattice constant and radius of the cavities for $\mathrm{LN}$. The steady state electric field figure for the TE-polarized wave propagation for silicon through an optimized splitter configuration at $1.55 \mu \mathrm{m}$ wavelength where the wavelength range is between 1.3-1.98 $\mu \mathrm{m}$ and the band gap for the combiner of the MZI modulator with the same of lattice constant and radius of the cavities. 
Figure 7 shows the result of the untapered of PCW with all same radius of the holes where $\mathrm{R}=0.192$ for $\mathrm{LN}$ and $\mathrm{R}=0.2$ for the silicon in the wavelength range $(1.53 \mu \mathrm{m}-1.57 \mu \mathrm{m})$. The optimization applied the gaussian modulated continuous wave of the input field tranverse. The total efficiency of the output splitter and combiner for the LN material are $65 \%$ and $18 \%$ and the total efficiency of the output for the splitter and combiner for the silicon material are $40 \%$ and $4 \%$ at $1.55 \mu \mathrm{m}$ operating wavelength. This means that the output efficiency on the combiner for the silicon is higher compared to LN due to the loss of the wave propagation through the MZI modulator waveguide. The radius of all holes for both LN and silicon materials are fixed as mentioned in Figure 6.

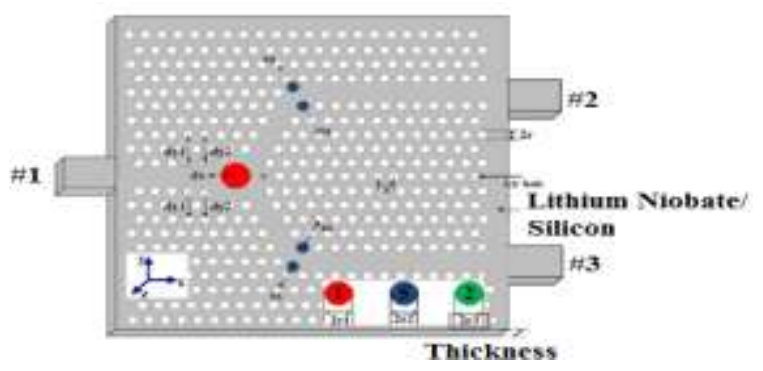

Figure 5. The optimized schematic design of the PCW splitter-combiner MZI modulator

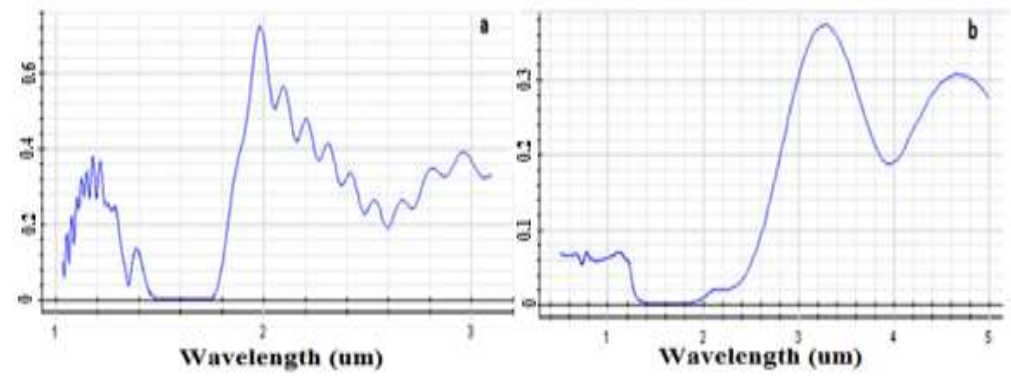

(b)

Figure 6. The band diagram and transmisson for the (a) LN and (b) for the silicon materials
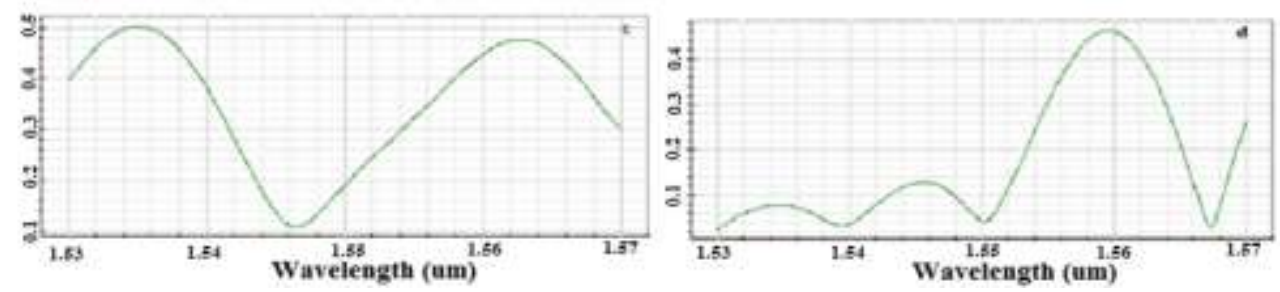

(a)
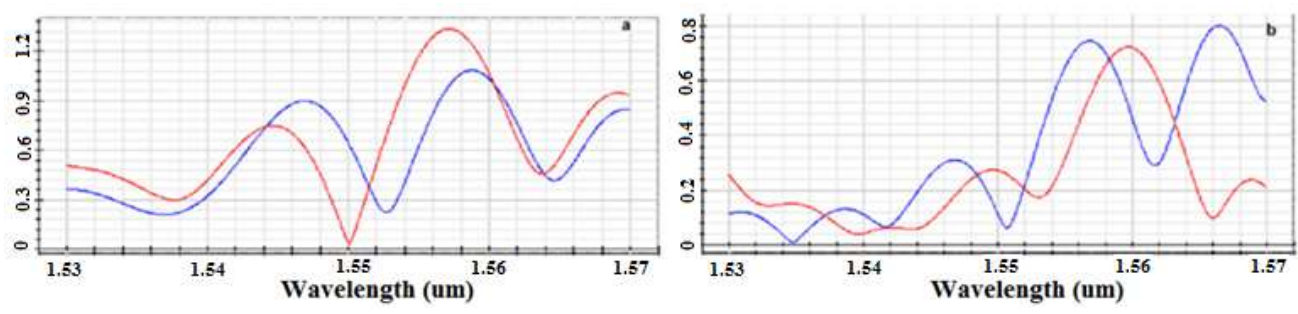

(b)

Figure 7. The transmission efficiency performance for the splitter of LN and silicon (a) and (b). The transmission efficiency performance for the combiner of $\mathrm{LN}$ and silicon (c) and (d) 
Figure 8 shows the results of tapered PCW transmission for the optimized splitter and combiner in the wavelength range $(1.53 \mu \mathrm{m}-1.57 \mu \mathrm{m})$. The total efficiency of the output splitter and combiner for the $\mathrm{LN}$ material are $50 \%$ and $89 \%$ and the total transmission of the output splitter and combiner compared to the previous research are $40 \%$ and $70 \%$ and for the silicon material are achieved $55 \%$ and $18 \%$ at $1.55 \mu \mathrm{m}$ operating wavelength. This means that the output transmission at the combiner for the $\mathrm{LN}$ is higher compare to the silicon due to the loss of the wave propagation through the mzi modulator waveguide when the parameters of the designated cavities are defined $\mathrm{r} 1=0.763 \mathrm{a}, \mathrm{r} 5=0.436 \mathrm{a}, \mathrm{r} 2=0.218 \mathrm{a}$, dy $1=0.109 \mathrm{a}$, and dy2= $0.218 \mathrm{a}$. Then the paramerters numbered holes for silicon are $\mathrm{r} 1=0.6 \mathrm{a}, \mathrm{r} 5=0.36 \mathrm{a}, \mathrm{r} 2=0.2616 \mathrm{a}, \mathrm{dy} 1=0.1308 \mathrm{a}$, and dy $2=0.2616 \mathrm{a}$. Then Table 1 shows the comparison of LN and silicon MZI modulator.
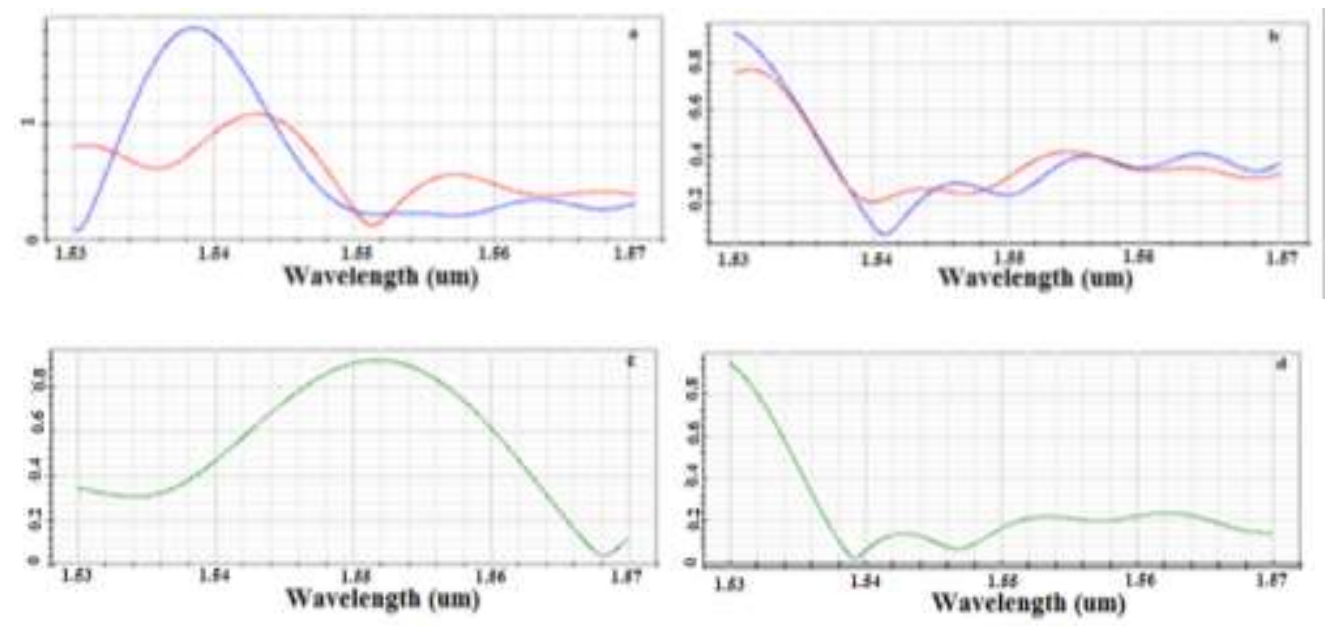

Figure 8. The calculated transmission efficiency performance for the splitter of LN and silicon (a) and (b). The transmission efficiency performance for the combiner of LN and silicon (c) and (d)

Table 1. The comparison of Lithium Niobate and Silicon MZI modulator

\begin{tabular}{|c|c|c|c|}
\hline Materials & Group velocity $\left(\mathrm{V}_{\mathrm{g}}\right)$ & Calculation & Efficiency (\%) \\
\hline Lithium Niobate & $\begin{array}{l}\text { depends on the change of the } \\
\text { refractive index where (Refractive } \\
\text { index, RI vs. Voltage) }\end{array}$ & $\begin{aligned} \Delta n & =-\frac{n^{3} L N \gamma_{33} U}{2 d} \\
& \frac{2 \pi}{\lambda} \cdot \Delta n \cdot L\end{aligned}$ & $\begin{array}{l}\text { The percentage of the output splitter and } \\
\text { combiner for the LN is } 65 \% \text { and } 18 \% \text { of } \\
\text { untapered PCW. Then the percentage of } \\
\text { the output splitter and combiner for the } \\
\text { LN is } 50 \% \text { and } 89 \% \text { of tapered PCW. }\end{array}$ \\
\hline Silicon & $\begin{array}{l}\text { depends on the carrier concentration } \\
\text { of holes and electrons }\end{array}$ & $\begin{array}{c}\Delta \mathrm{n}=\Delta \mathrm{ne}+\Delta \mathrm{nh}=- \\
{\left[8.8 \times 10^{-}\right.} \\
{ }^{22}(\Delta \mathrm{Ne})+8.5 \times 10^{-} \\
\left.{ }^{18}(\Delta \mathrm{Nh}) 0.5\right] \\
\quad \frac{2 \pi}{\lambda} \cdot \Delta n . L \\
\Delta \emptyset=\end{array}$ & $\begin{array}{l}\text { The percentage of the output splitter and } \\
\text { combiner for the silicon is } 40 \% \text { and } 4 \% \\
\text { of untapered PCW. Then the percentage } \\
\text { of the output splitter and combiner for } \\
\text { the silicon is } 55 \% \text { and } 18 \% \text { of tapered } \\
\text { PCW. }\end{array}$ \\
\hline
\end{tabular}

\section{CONCLUSION}

The study is related to the comparison of the performances on different materials MZI modulator which are LN and silicon material. Modulator perfoirmance is studied on a photonic crystal waveguide based on optimization using the OptiWave software. The MZI modulator of LN gives the highest phase shift which is $2.45784 \mathrm{rad}$ and it gives the better performance and the attain the set of slow light waveguides that supports a low group velocity over a flat signal group velocity region. The percentage of the splitter is $50 \%$ and combiner is $89 \%$ optimized MZI modulator design LN material used. With tapered techniques or methods, the transmission is greatly improved about $70 \%$ for the LN MZI modulator compared to the previous research for the photonic device application based on the photonic crystal properties and design.

\section{ACKNOWLEDGEMENTS} Shah Alam.

The author wish to thank to IPSIS, GOEE and Faculty of Electrical Engineering, FKE of UiTM 


\section{REFERENCES}

[1] C. C. Architect, "On-Chip On-Chip Photonic Photonic Interconnects Interconnects," 2013.

[2] L. Lentine and C. T. DeRose, "Challenges for optical interconnect for beyond Moore's law computing," 2016 IEEE Int. Conf. Rebooting Comput. ICRC 2016-Conf. Proc., pp. 2-5, 2016, doi: 10.1109/ICRC.2016.7738696.

[3] T. Kawanishi, K. Inagaki, A. Kanno, and N. Yamamoto, "Precise measurement techniques for optical-to-electric conversion devices," vol. 9, pp. 3906-3906, 2016.

[4] S. Almosni et al., "Material challenges for solar cells in the twenty-first century: directions in emerging technologies," Sci. Technol. Adv. Mater., vol. 19, no. 1, pp. 336-369, 2018, doi: 10.1080/14686996.2018.1433439.

[5] M. A. Green and S. P. Bremner, "Energy conversion approaches and materials for high-efficiency photovoltaics," Nat. Mater., vol. 16, no. 1, pp. 23-34, 2016, doi: 10.1038/nmat4676.

[6] X. Wang and B. Sensale-rodriguez, "Learn more about Optical Modulation Graphene Based Optical Interconnects," 2019.

[7] Boes, B. Corcoran, L. Chang, J. Bowers, and A. Mitchell, "Status and Potential of Lithium Niobate on Insulator (LNOI) for Photonic Integrated Circuits," Laser Photonics Rev., vol. 12, no. 4, pp. 1-19, 2018, doi: 10.1002/lpor.201700256.

[8] M. Thomaschewski, V. A. Zenin, C. Wolff, and S. I. Bozhevolnyi, "Plasmonic monolithic lithium niobate directional coupler switches," Nat. Commun., vol. 11, no. 1, pp. 1-19, 2020, doi: 10.1038/s41467-020-14539-y.

[9] P. Sciences and E. Accelerator, "Integrated modulator is now better than ever, made by the best optical materials," no. September, pp. 2018-2020, 2018.

[10] C. Wang, M. Zhang, X. Chen, M. Bertrand, A. Shams-Ansari, S. Chandrasekhar, P. Winzer, and M. Lončar, "Integrated lithium niobate electro-optic modulators operating at CMOS-compatible voltages," Nature, vol. 562, no. 7725, pp. 101-104, 2018.

[11] M. Xu et al., "High-performance coherent optical modulators based on thin-film lithium niobate platform," Nat. Commun., vol. 11, no. 1, pp. 1-7, 2020, doi: 10.1038/s41467-020-17806-0.

[12] D. Marpaung, J. Yao, and J. Capmany, "Integrated microwave photonics," Nat. Photonics, vol. 13, no. 2, pp. 80-90, 2019, doi: 10.1038/s41566-018-0310-5.

[13] G. S. Jeong, W. Bae, and D. K. Jeong, "Review of CMOS integrated circuit technologies for High-Speed photodetection," Sensors (Switzerland), vol. 17, no. 9, 2017, doi: 10.3390/s17091962.

[14] S. Gupta, W. T. Navaraj, L. Lorenzelli, and R. Dahiya, "Ultra-thin chips for high-performance flexible electronics," NPJ Flex. Electron., vol. 2, no. 1, 2018, doi: 10.1038/s41528-018-0021-5.

[15] Y. Koga and K. Kurita, "Fabrication of silicon on insulator wafer with silicon carbide insulator layer by surfaceactivated bonding at room temperature," Jpn. J. Appl. Phys., vol. 59, no. 5, 2020, doi: 10.35848/1347-4065/ab82af.

[16] G. W. Lu et al., "High-temperature-resistant silicon-polymer hybrid modulator operating at up to $200 \mathrm{Gbit} s-1$ for energy-efficient datacentres and harsh-environment applications," Nat. Commun., vol. 11, no. 1, pp. 1-9, 2020, doi: 10.1038/s41467-020-18005-7.

[17] S. Koeber, R. Palmer, M. Lauermann, W. Heni, D. L. Elder, D. Korn, M. Woessner, L. Alloatti, S. Koenig, P. C. Schindler, H. Yu, W. Bogaerts, L. R. Dalton, W. Freude, J. Leuthold, and C. Koos, "Femtojoule electro-optic modulation using a silicon-organic hybrid device," Light Sci. Appl., vol. 4, no. 2, 2015.

[18] H. Mahrous, M. Fedawy, M. El Sabbagh, W. Fikry, and M. Gad, "Design of a $90 \mathrm{GHz}$ SOI fin electro-optic modulator for high-speed applications," Appl. Sci., vol. 9, no. 22, 2019, doi: 10.3390/app9224917.

[19] J. Witzens, "High-Speed Silicon Photonics Modulators," Proc. IEEE, vol. 106, no. 12, pp. 2158-2182, 2018, doi: 10.1109/JPROC.2018.2877636.

[20] M. Irfan Anis, "All Optical Signal Processing Technologies in Optical Fiber Communication," Opt. Fiber Appl., pp. 1-26, 2020, doi: 10.5772/intechopen.88354.

[21] J. Yu and J. Zhang, "Recent progress on high-speed optical transmission," Digit. Commun. Networks, vol. 2, no. 2, pp. 65-76, 2016, doi: 10.1016/j.dcan.2016.03.002.

[22] R. P. Dionisio, "Interferometry Applications in All-Optical Communications Networks," Opt. Interferom., 2017, doi: $10.5772 / 66133$.

[23] P. Minzioni, C. Lacava, T. Tanabe, J. Dong, X. Hu, G. Csaba, W. Porod, G. Singh, A. E. Willner, A. Almaiman, V. Torres-Company, J. Schröder, A. C. Peacock, M. J. Strain, F. Parmigiani, G. Contestabile, D. Marpaung, Z. Liu, J. E. Bowers, L. Chang, S. Fabbri, M. Ramos Vázquez, V. Bharadwaj, S. M. Eaton, P. Lodahl, X. Zhang, B. J. Eggleton, W. J. Munro, K. Nemoto, O. Morin, J. Laurat, and J. Nunn, "Roadmap on all-optical processing," J. Opt. (United Kingdom), vol. 21, no. 6, 2019.

[24] N. S. Hidayah, "Performance of different Mach-Zehnder interferometer (MZI) structures for optical modulator," $J$. Telecommun. Electron. Comput. Eng., vol. 9, no. 2-8, pp. 25-29, 2017.

[25] Y. Zhang, H. Tian, D. Yang, H. Liu, Y. Liu, and Y. Ji, "Ultra-compact low-voltage and slow-light MZI electrooptic modulator based on monolithically integrated photonic crystal," Opt. Commun., vol. 315, pp. 138-146, 2014, doi: 10.1016/j.optcom.2013.10.020.

[26] Lotfian, R. Yadipour, and H. Baghban, "Broadrange tunable slow and fast light in quantum dot photonic crystal structure," Chinese Phys. B, vol. 26, no. 12, pp. 1-7, 2017, doi: 10.1088/1674-1056/26/12/124207.

[27] T. Qin et al., "Fast- and slow-light-enhanced light drag in a moving microcavity," Commun. Phys., no. 2020, pp. 18, doi: 10.1038/s42005-020-0386-3.

[28] Y. Liu and C. Jiang, "Dispersionless one-way slow wave with large delay bandwidth product at the edge of gyromagnetic photonic crystal," Int. J. Mod. Phys. B, vol. 34, no. 10, pp. 1-10, 2020, doi: 10.1142/S0217979220500861. 
[29] M. Passoni, D. Gerace, L. O’Faolain, and L. C. Andreani, "Slow light with interleaved p-n junction to enhance performance of integrated Mach-Zehnder silicon modulators," Nanophotonics, vol. 8, no. 9, pp. 1485-1494, 2019, doi: 10.1515/nanoph-2019-0045.

\section{BIOGRAPHIES OF AUTHORS}

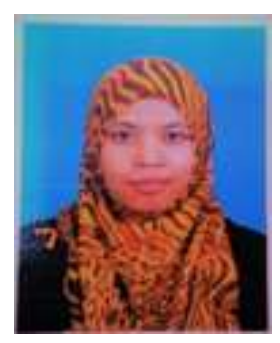

Nor Hidayah Binti Roslan received MSc. in electrical engineering (Communication) in 2017. $\mathrm{PhD}$ student at Universiti Teknologi MARA (UiTM) in Faculty of Electrical Engineering (Communication). Her research in Photonics and focus on the simulations and analysis of the photonic crystal cavities and the Mach-Zehnder Interferometer (MZI) modulator by using OptiWave and Lumerical software. She has published article in the Journal of Electrical and Electronic System Research.

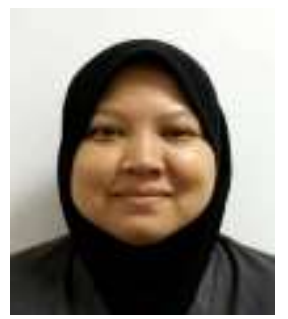

Aziati H. Awang received the B. Eng (Hons) from UNITEN, in 2000, MSc. In Personal. Mobile and Satellite Engineering from University of Bradford in 2003 and $\mathrm{PhD}$ in Terahertz Technologies from University of Leeds in 2013. She is actively engaged in the Photonics \& Microwave devices and IOT based research. She is currently a Senior Lecturer in the Centre for Communication Engineering Studies, Electrical Engineering Faculty, Universiti Teknologi MARA (UiTM). She is also a member of Institute Electrical and Electronics Engineering (IEEE) and Institution of Engineering and Technology (IET).

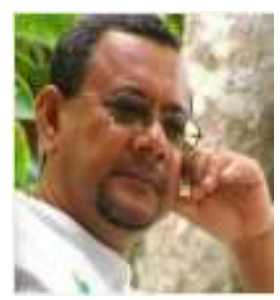

Mohd Hanapiah Mohd Yusoff received the B.Sc. (Hons.) degree in Physics from the University of Liverpool, U.K., in 1983, the M.Sc. (Distinction) degree in Microwave Solid State Physics from the University of Portsmouth, U.K., in 1994, and the Ph.D. degree in the field of Optical Communications from the School of Physics, Universiti Sains Malaysia, in 2010. His working experience includes teaching at Universiti Teknologi Malaysia, Universiti Putra Malaysia, Universiti Teknologi MARA and also as a Geophysical Engineer with Schlumberger Overseas S.A. He is involved in the investigation and analysis of tapered fiber and photonic crystal fiber sensors. His research interest is in the lightwave propagation in planar photonic devices with emphasis on photonic band gap structures and slotted silica waveguide structures.

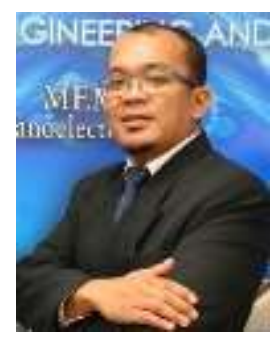

Ahmad Rifqi Md Zain is Associate professor of Electronics engineering at Institute of Microengineering and Nanoelectronics (IMEN), UKM. He received his deg and masters from Coventry University and Glasgow university, UK in 2002 and 2005. In 2009, he received his $\mathrm{PhD}$ from Glasgow University, UK (with Prof Richard De La Rue), in Electronins engineering (1D photonic crystal cavities). After completing his postdoctoral studies in 2012 at Bristol university in Gallium nitride based optical biosensors (with Prof Martin J. Cryan), he joined IMEN, in 2014. In 2017, he joined as a postdoctoral research fellow in applied physics until 2019, working on Quantun information. He is an expert in nanophotonics, optoelectronics and nanofabrication, and his current interest include optical imaging, 2D materias, optical based biosensors, 1D and 2D photonic crystals, opical MEMS sensors. 